\title{
Vocablos relacionados con el recurso micológico en el idioma de la cultura chinanteca de la Sierra Norte del estado de Oaxaca, México
}

\section{Vocabulary related to the mycological resource in the Chinantec culture from the Northern Mountain Range of the state of Oaxaca, Mexico}

\author{
Alexanders López García', Mario Jiménez-Ruiz', Jesús Pérez-Moreno²
}

'Universidad de la Sierra Juárez, Instituto de Estudios Ambientales, Av. Universidad S/N, Ixtlán de Juárez, Oaxaca, México. ${ }^{2}$ Colegio de Postgraduados, Campus Montecillo. Microbiología, Edafología. Km. 36.5 carretera México-Texcoco, Montecillo, Estado de México, México.

Jesús Pérez Moreno, e-mail: jperezm@colpos.mx

\section{RESUMEN}

Antecedentes: El uso de los hongos se remonta a miles años, evidencia de ello es el gran número y variedad de nombres asignados a especies de importancia cultural. A través de estos nombres es posible entender el marco cognitivo-conceptual de los hongos existente en los diferentes grupos humanos.

Objetivo: Documentar el conocimiento tradicional relacionado con el origen y significado de los nombres de los hongos utilizados actualmente por la cultura chinanteca la cual habita en Oaxaca en el sureste de México.

Métodos: Mediante entrevistas no estructuradas, semiestructuradas y estructuradas se obtuvieron los vocablos chinantecos relacionados con el uso tradicional de los hongos en dos comunidades del Estado de Oaxaca. Asimismo se realizó la traducción de cada vocablo y se buscó su etimología y posible origen.

Resultados y conclusiones: Se presenta un listado de 70 vocablos relacionados con los hongos silvestres utilizados por los chinantecos con uso comestible, medicinal, lúdico y ornamental. Todos los vocablos registrados tienen una traducción al español y se encuentran relacionados con 36 especies fúngicas. Este artículo documenta por primera ocasión la gran riqueza lingüística y conocimiento tradicional de los hongos, el cual ha pervivido, en la cultura chinanteca.

Palabras clave: importancia biocultural, conocimiento tradicional, hongos comestibles, etnomicología.

\section{ABSTRACT}

Background: Humans have used the mushrooms during thousands of years as evidenced by the great number and variety of names assigned to species of cultural importance. Through these names it is possible to understand the cognitive-conceptual framework of mushrooms existing in different human groups.

Objective: To document traditional knowledge related to the origin and meaning of the names of the mushrooms currently used by the Chinantec culture which inhabit in the state of Oaxaca in southeastern Mexico.

Methods: Through non-structured and semi-structured interviews, the Chinantec words used to designate mushrooms were obtained in two communities of the State of Oaxaca. Also the translation of each word was carried out and its etymology and possible origin were investigated.

Results and conclusions: A list of 70 words related to the wild mushrooms used by the Chinantec people with edible, medicinal, ludic and ornamental use is presented. All the registered words have a translation into Spanish and are related to 36 fungal species. This article documents for the first time the great linguistic richness and traditional knowledge of mushrooms, which has survived, in the Chinantec culture.

Keywords: biocultural importance, traditional knowledge, edible mushrooms, ethnomycology. 


\section{INTRODUCCIÓN}

La riqueza biocultural presente no solo en México sino en todo el mundo, ha contribuido a que los diferentes grupos humanos hayan adoptado a lo largo del tiempo diferentes percepciones y usos sobre el aprovechamiento de los recursos naturales (RuanSoto et al., 2009). Tal es el caso de los hongos silvestres, que son un recurso forestal no maderable que ha generado un gran impacto sobre los grupos culturales del mundo, incluyendo principalmente su importancia como alimento, medicina, uso recreativo y lúdico.

Además de tener una gran importancia ecológica, ya que más de 20,000 especies de hongos se encuentran asociados simbióticamente con más de 5,000 especies de árboles de importancia forestal, formando ectomicorrizas, y actualmente se está iniciando el desarrollo biotecnológico para la conservación de los bosques haciendo uso de esta simbiosis (Pérez-Moreno, 2012).

Adicionalmente, estudios recientes de macromicetos han demostrado su gran valor nutracéutico y contenido de compuestos bioactivos con propiedades medicinales (Pérez-Moreno y Martínez-Reyes, 2014).

México alberga 64 grupos étnicos (INI, 2003) y cuenta con 61 lenguas y 291 variantes dialectales (Gordon, 2005). La mayor parte de estos grupos étnicos del país poseen un alto conocimiento tradicional sobre el uso de los hongos silvestres comestibles (Guzmán, 2011).

Uno de estos grupos es la etnia chinanteca, que se ubica en el la Sierra Norte de Oaxaca en los límites con el estado de Veracruz; cuenta con 138, 741 habitantes (INEGI, 2015) y su lengua chinanteca es uno de los 14 idiomas indígenas del estado de Oaxaca que data de más de 3500 años, y es derivada de la lengua llamada otomangue la primera lengua del estado de Oaxaca, actualmente extinta (Barbaras y Bartolomé, 1990).

A pesar de su gran relevancia, actualmente la importancia de los hongos en el idioma chinanteco ha recibido escasa atención. El presente estudio registra un listado de 70 vocablos relacionados con el uso y percepción de los habitantes chinantecos relacionado con el recurso micológico.

Cada uno de los vocablos presenta su traducción al castellano, así como la interpretación de dichos términos de acuerdo a la percepción de los pobladores locales.

\section{MATERIALES Y MÉTODOS}

Las comunidades estudiadas en el presente trabajo fueron la agencia de La Esperanza, Comaltepec y la cabecera municipal de Santiago Comaltepec, pertenecientes al distrito de Ixtlán de Juárez, del estado de Oaxaca. El municipio de Santiago Comaltepec, se encuentra ubicado en la Chinantla Alta entre las coordenadas geográficas $96^{\circ} 32^{\prime} 54^{\prime \prime}$ de longitud oeste y $17^{\circ} 33^{\prime} 54^{\prime \prime}$ de latitud norte (Figura 1). Colinda al norte con los municipios de San Juan Quiotepec, San Pedro Yolox y San Juan Bautista Valle Nacional; al sur con San Pablo Macuiltianguis e Ixtlán de Juárez, al oeste con San Pablo Macuiltianguis y San Pedro Yolox; al este con Ayotzintepec e Ixtlán de Juárez (CONAPO, 1994). Los tipos de vegetación que predominan en el municipio de Santiago Comaltepec son: i) bosque de coníferas con clima templado-húmedo y especies arbóreas representativas de Pinus patula, P. ayacahuite, P. teocote, P. pseudoestrobus, P. oocarpa, P. montezumae, Quercus crassifolia y Q. elliptica; y ii) bosque mesófilo de montaña con clima templado-húmedo y vegetación representativa de Liquidambar styraciflua y Quercus spp. con presencia de especies de las familias Orquidaceae, Bromeliaceae y Piperaceae (Rzedowski, 2006). El municipio de Santiago Comaltepec (Figuras 2 a, b) cuenta con una población aproximada de 1115 habitantes de los cuales el $43 \%$ son hombres y el $57 \%$ son mujeres. La comunidad de Santiago Comaltepec alberga 710 habitantes, mientas que la comunidad de la esperanza cuenta con 128 habitantes (INEGI, 2010).

Se realizaron visitas a la comunidad de Santiago Comaltepec y la Esperanza Comaltepec durante los meses de mayo a octubre de 2014, 2015. Durante la temporada de lluvia y la época de recolección de hongos comestibles, se entrevistaron a las personas de ambas comunidades. Las entrevistas se realizaron dos veces al mes en cada una de las comunidades. Se realizaron encuestas al $13 \%$ de un total de 305 familias presentes en ambas comunidades, tomando en cuenta los criterios de BurrolaAguilar et al. (2012) y Domínguez-Romero et al. (2015). Las viviendas fueron seleccionadas al azar, sin embargo, cuando el informante estaba encubierto desde el punto de vista externo, entonces se acudió al muestreo de "bola de nieve" (Sandoval, 2002). En los hogares se realizaron encuestas a las personas que se dedican a la recolección de hongos silvestres. Se efectuaron preguntas no estructuradas, semiestructuradas y estructuradas 


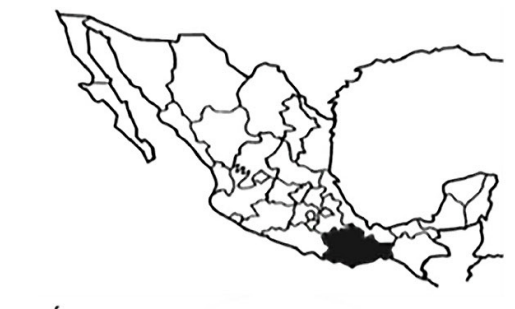

MÉXICO

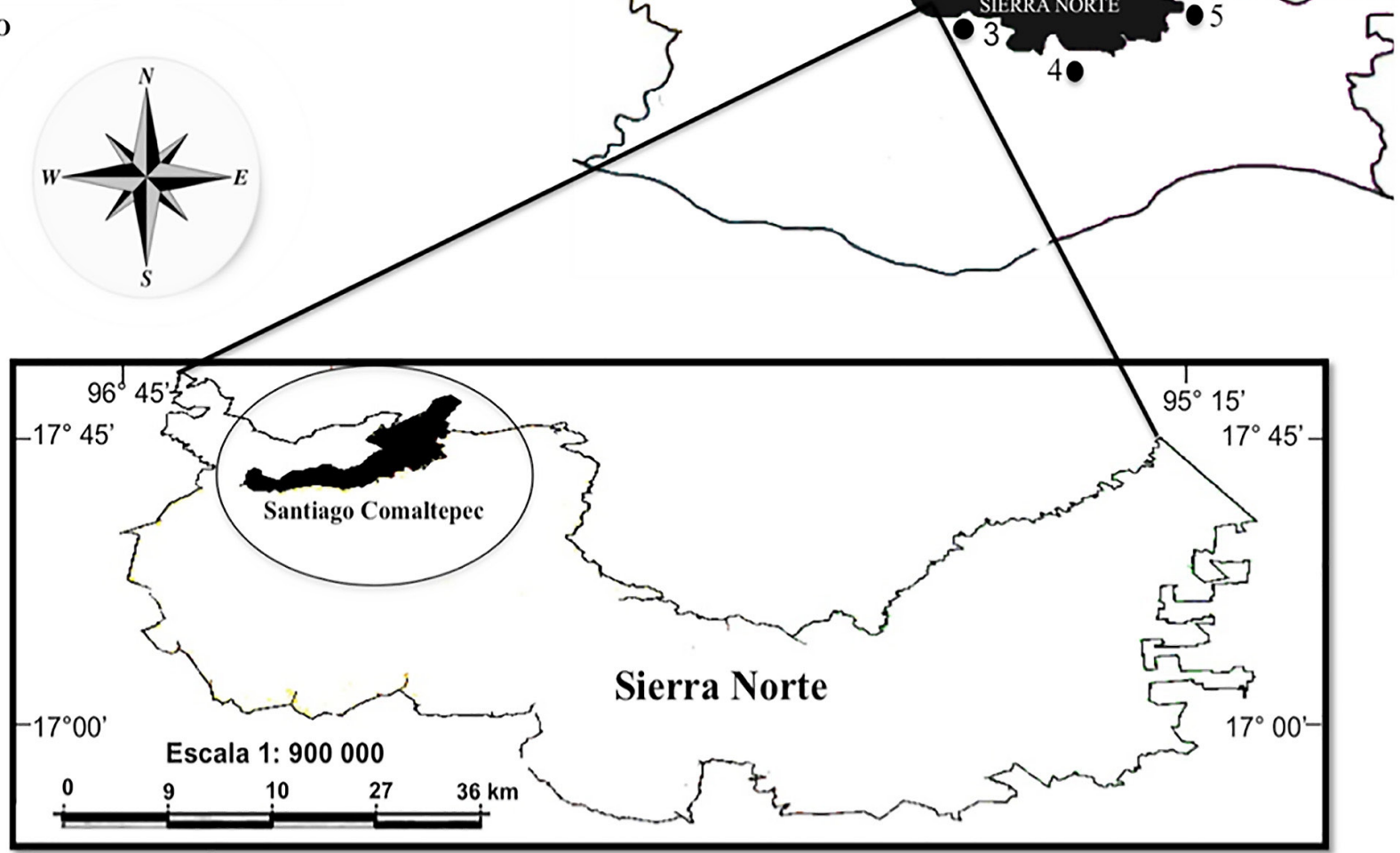

Figura 1. Ubicación geográfica del municipio chinanteco de Santiago Comaltepec, Ixtlán, Oaxaca. 1) región del Papaloapan; 2) región de la Cañada; 3) región de los Valles Centrales; 4) región de la Sierra Sur; y 5) región del Istmo.

con la finalidad de obtener información acerca de los vocablos relacionados con el conocimiento prevaleciente del recurso micológico, incluyendo nombres de los hongos, usos e importancia de los hongos silvestres (comestible, lúdico, medicinal, mágico o tóxico), modos de preparación, así como aspectos ecológicos y de las partes de los hongos en lenguaje nativo siguiendo los métodos propuestos por Ruan-Soto et al., 2004; GaribayOrijel et al., 2006; Burrola-Aguilar et al., 2012).

Para la escritura y significado de los nombres en chinanteco, se realizó investigación bibliográfica y se buscó el apoyo de traductores bilingües locales. Se utilizó el vocabulario chinanteco-español de Santiago Comaltepec (Anderson y Goodwin, 2007), así como, vocabularios de otras comunidades chinantecas (Skinner y Skinner, 2000; Rupp et al., 2012). Para reconocer la identidad de los hongos en el momento de las entrevistas se utilizaron álbumes fotográficos de especies de hongos de la región y en ocasiones se utilizaron especímenes frescos previamente recolectados en campo. La identificación fúngica se llevó a cabo utilizando técnicas micológicas convencionales, las cuales consistieron en efectuar una caracterización macromorfológica de los esporomas, tomando en consideración caracteres tales como tamaño, color, textura, forma o tipo de himenio; y micromorfológica evaluando la forma, tamaño y ornamentación de las esporas y de estructuras específicas como basidios, cistidios o presencia de fíbulas. Para la identificación de las especies se utilizaron publicaciones especializadas (Arora, 1986; Guzmán, 1983; Guzmán y Ramírez-Guillén, 2001; Trudell y Ammirati, 2009). 


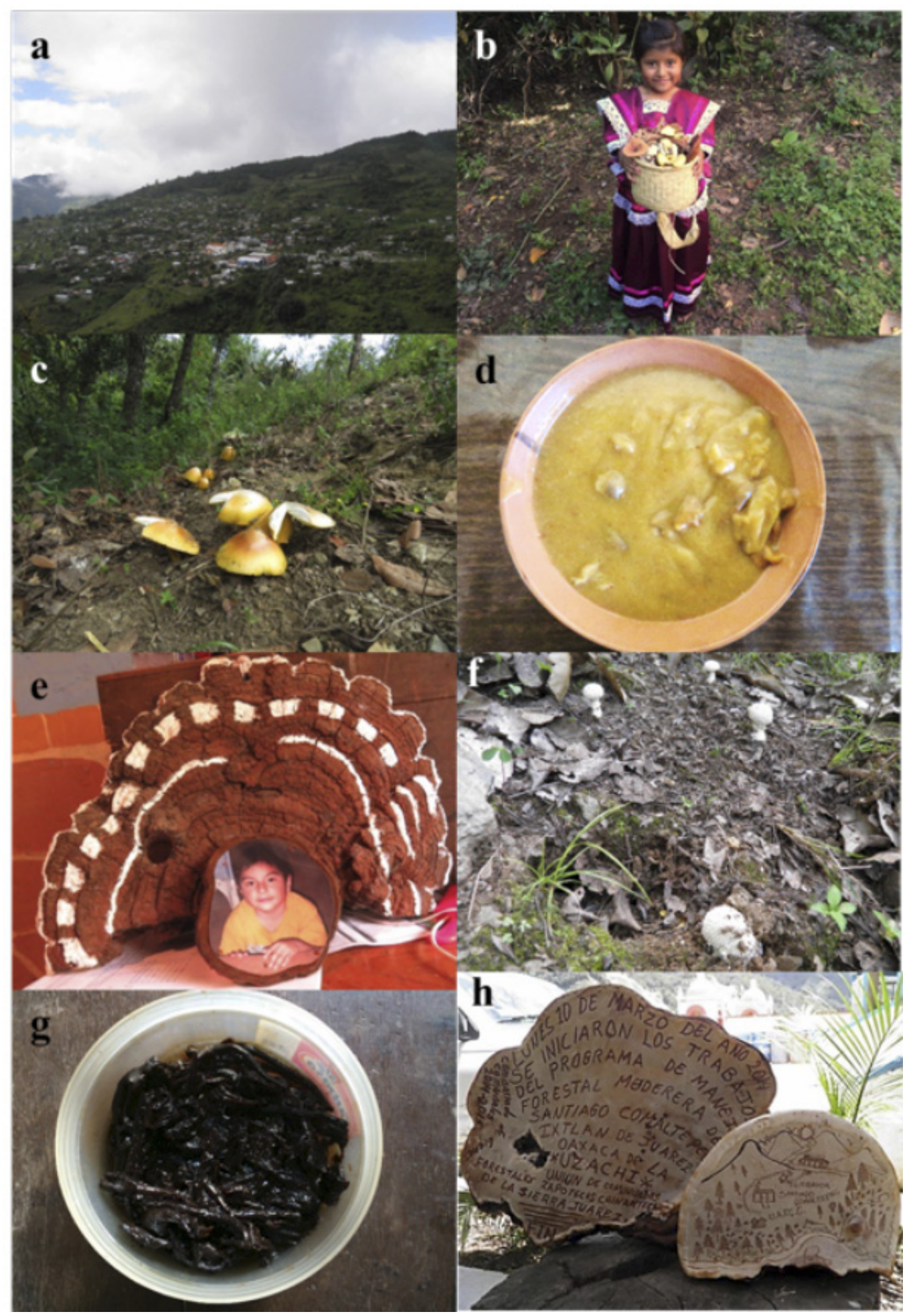

Figura 2. Uso de los hongos silvestres comestibles en la comunidad chinanteca de Santiago Comaltepec, Oaxaca. a) Vista panorámica de Santiago Comaltepec. b) Niña chinanteca sosteniendo un tenate con hongos comestibles. c) Amanita basii Guzmán et Ram.-Guill. en bosque de Quercus spp., su hábitat natural. d) “Amarillo de hongos”, el platillo más valorado en Santiago Comaltepec. e) Portarretratos hecho con Ganoderma sp., el cual se pinta y asemeja a la cola de un guajolote. f) Amanita sp. con escamas piramidales, en bosque de encino, considerada tóxica en la comunidad. g) Hongos alucinógenos en miel utilizados en la medicina tradicional chinanteca. h) Frases y dibujos realizados en el himenio de Ganoderma applanatum (Pers.) Pat., el cual se encuentra en las oficinas del comisariado de bienes comunales de la localidad de estudio. 
Se hicieron recorridos exhaustivos a 15 parajes del municipio para realizar la recolección de hongos silvestres y su posterior herborización, para ser depositados en la colección de la Universidad de la Sierra Juárez, en Ixtlán de Juárez, Oaxaca. Los recorridos se realizaron en compañía de los recolectores previamente entrevistados que presentaban un amplio conocimiento sobre la identificación y usos de los hongos silvestres, esto con la finalidad de confirmar la identidad y determinación taxonómica de los hongos mencionados en las entrevistas. Un factor que facilitó la recopilación de la información fue el hecho de que el primer autor es hablante nativo de chinanteco y originario de la comunidad de estudio.

\section{Resultados}

Se registraron 70 vocablos relacionados con los hongos de importancia para los habitantes de la población estudiada. Todos los vocablos registrados fueron traducidos al español, se encuentran relacionados con 36 especies fúngicas (Tabla 1) y la mayoría se relacionan con especies de hongos comestibles (82\%) y en menor grado con uso lúdico $(6 \%)$, uso medicinal $(6 \%)$ y especies tóxicas $(6 \%)$. Todos los vocablos hacen referencia a ciertas particulares de cada especie de hongo, abarcando desde la forma de crecimiento, el color, la forma, la textura, los usos, e incluso homologías con objetos relacionados con la cosmovisión de la cultura chinanteca incluyendo alusiones a aspectos religiosos. Un factor que resultó de enorme importancia en el grado de reconocimiento de especies fue la edad, dado que las personas de mayor edad fueron capaces de distinguir un mayor número de especies de hongos (Tabla 2). Los vocablos registrados relacionados con el recurso micológico en la zona de estudio fueron los siguientes:

Cuinii: encino, sufijo utilizado para nombrar especies de hongos que crecen asociados a dichos árboles del género Quercus tales como Hebeloma fastibile (Pers.) P. Kumm. o Amanita jacksonii Pomerl (Figura 2c).

Cha'nit': hongo. Término utilizado por los chinantecos de Ayotzintepec, Ozumacín y Progreso para el universo de los hongos (Rupp et al., 2012).

Dséc cui: hijo o retoño de la milpa, término utilizado para nombrar al hongo parásito del maíz Ustilago maydis (DC) Corda el cual es considerado comestible.
Dsia laan: brujo o chamán, persona que hace uso de los hongos medicinales.

Dsia: persona, sufijo utilizado para designar especies de hongos que presentan alguna homología con las partes del cuerpo de una persona por ejemplo con una oreja en el caso de Auricularia spp.

Dsií: gusano, vocablo utilizado como sufijo para nombrar los dientes del himenio de especies del género Hydnum (tales como H. repandum L. o H. rufescens Pers.), debido a que asemejan a pequeños gusanos que infectan principalmente el intestino delgado y son conocidos por los pobladores por su presencia en el excremento de los humanos.

Dsit: vara, sufijo utilizado para nombrar especies de hongos con forma de la vara o rama de un árbol, por ejemplo especies del género Ramaria.

E $j l^{\prime `}$ : más sabroso, vocablo utilizado para las especies con mayor índice de preferencia entre los chinantecos de Santiago Comaltepec, por ejemplo Neolentinus suffrutescens (Brot.) T.W. May \& A.E. Wood

Fii: leche, término utilizado como sufijo para designar hongos que secretan látex, denominada leche por los pobladores locales, por ejemplo Lactarius volemus (Fr.) Fr.

Gmig: caldo o guisado, nombre del platillo elaborado a base de puré de tomate, chile, cebolla y ajo, en el cual se le agregan los hongos precocidos. Esta forma de preparación de los hongos es también muy apreciada entre los chinantecos.

Güí: humedad, término utilizado para nombrar sitios con alta humedad, en donde los habitantes mencionan hay mayor abundancia de hongos.

Guñiá: relámpago, ráfaga de luz con un estruendo emergido del cielo que indica el comienzo de la temporada de hongos.

Jäá: espuma, término utilizado para designar a los mohos (hongos filamentosos) que crecen sobre los alimentos.

Jgo: podrido, término para nombrar especies de hongos que están en proceso de descomposición o que tienen una vida de anaquel muy corta por ejemplo Suillus granulatus (L.) Roussel.

Jii 'jmíi: temporada de lluvias, meses del año que corresponden de abril a octubre, donde los habitantes de la comunidad salen a recolectar hongos en el bosque. 
Tabla 1. Hongos silvestres utilizados en la comunidad chinanteca de Santiago Comaltepec, Oaxaca y sus nombres y usos.

\begin{tabular}{|c|c|c|c|}
\hline Nombre científico & Nombre en chinanteco & Nombre común & Uso \\
\hline Agaricus campestris L. & Nít kuän ni jmiï jó & Hongo que crece sobre estiércol de ganado & $C, A C$ \\
\hline Amanita basii Guzmán \& Ram.-Guill. & Nít kua' jnéë & Hongo bendito amarillo & $C, A C, C O C$ \\
\hline Amanita jacksonii Pomerl & Nît kua' yöö & Hongo bendito rojo & $C, A C, C O C$ \\
\hline Amanita laurae Guzmán \& Ram.-Guill. & Nît kua’ jnéë & Hongo bendito amarillo & $C, A C, C O C$ \\
\hline Auricularia delicata (Mont. ex Fr.) Henn. & Nít loguaạ dsia ri & Hongo oreja de duende & $C, A C$ \\
\hline Auricularia polytricha (Mont.) Sacc. & Nít loguaạ dsia ri & Hongo oreja de duende & $C, A C$ \\
\hline Auricularia sp. Bull. & Nít loguaạ dsia ri & Hongo oreja de duende & $C, A C$ \\
\hline Cantharellus cibarius Fr. & Nít jnéë & Hongo amarillo & $\mathrm{C}, \mathrm{AC}, \mathrm{COC}$ \\
\hline Craterellus tubaeformis (Fr.) Quél & Nît sdí guiị & Hongo de hiel de ardilla & $C, A C$ \\
\hline Favolus tenuiculus P. Beauv & Nít noöö & Hongo de manteca o de red & $C, A C$ \\
\hline Ganoderma applanatum(Pers.) Pat. & Nít óo & Hongo de viejo & L \\
\hline Ganoderma sp. P. Karst & Nít óo & Hongo de viejo & L \\
\hline Hebeloma fastibile (Pers.: Fr.) & Nít cuinií & Hongo de encino & $C, A C$ \\
\hline Hydnum repandum $\mathrm{L}$. & Nît dsii & Hongo de gusano & $C, A C$ \\
\hline Hydnum rufescens Schaeff. & Nî̀ dsii & Hongo de gusano & $C, A C$ \\
\hline Hygrophorus purpurascens (Alb. \& Schwein.) Fr. & Nít jnëøö & Hongo de ejote & $C, A C$ \\
\hline Hygrophorus russula (Schaeff.) Kauffman & Nít jnëøö & Hongo de ejote & $C, A C$ \\
\hline Hypomyces lactifluorum (Schwein.) Tul. \& C. Tul. & Nít uü & Hongo de chile & $C, A C$ \\
\hline Laccaria amethysteo-occidentalis G.M. Muell. & Nît tii tä’ & Hongo pata de pájaro & $C, A C$ \\
\hline Laccaria laccata var. pallidifolia (Peck) Peck. & Nít tii tä’ & Hongo pata de pájaro & $C, A C$ \\
\hline Laccaria nobilis A.H. Sm., Muell. & Nít tii tuziee & Hongo de pata de guajolote & $C, A C$ \\
\hline Laccaria trichodermophora G.M. Muell. & Nít tii tuziee & Hongo de pata de guajolote & $C, A C$ \\
\hline Lactarius volemus var. volemus (Fr.) Fr. & Nít fii & Hongo de leche & $\mathrm{C}, \mathrm{AC}, \mathrm{COC}$ \\
\hline Neolentinus suffrutescens (Brot.) T.W. May \& A.E. Wood & Nít kua' 'makíi & Hongo bendito de pino & $C, A C, C O C$ \\
\hline Pleurotus djamor (Rump. ex Fr.) & Nít 'majé & Hongo de jonote & $C, A C$ \\
\hline Pleurotus djamor var. roseus Corner & Nít 'matoo & Hongo de platanal & $C, A C$ \\
\hline Psilocybe yungensis Singer\& A.H. Sm. & Nít dsia jiun & Hongo enano & $C, M$ \\
\hline Psilocybe muliercula Sing. \& Smith. & Nít dsia jiun & Hongo enano & $C, M$ \\
\hline Ramaria sp. Holmsk. & Nït dsii & Hongo de vara & $C, A C$ \\
\hline Ramaria sp. Holmsk. & Nít dsii & Hongo de vara & $C, A C$ \\
\hline Ramaria sp. Holmsk. & Nít dsii & Hongo de vara & $C, A C$ \\
\hline Sparassis crispa (Wulfen) Fr. & Nít jniï & Hongo de nube o neblina & $C, A C$ \\
\hline Suillus granulatus L. & Nít tü’ ñii & Hongo baboso de zacate & $C, A C$ \\
\hline
\end{tabular}

Tabla 2. Grado del conocimiento micológico de los entrevistados de acuerdo a las edades y el número de especies que fueron capaces de identificar.

\begin{tabular}{ll}
\hline Rango de edades (años) & Grado de conocimiento* \\
\hline Menor de 35 & Medio \\
36 a 45 & Alto \\
46 a 60 & Muy alto \\
Mayor de 61 & Muy alto \\
\hline "Medio: 5 a 8 especies; Alto: 9 a 12 especies; Muy alto: más de 12 especies
\end{tabular}


Jiun: niño o pequeño, sufijo para nombrar especies alucinógenas por ejemplo Psilocybe muliercula Singer et A.H. Sm. y P. yungensis Singer et A.H. Sm., las cuales al ser consumidas originan eventualmente el encuentro con un enano (en otras culturas dichas criaturas son denominadas duendes), el cual contesta preguntas realizadas por la persona que consume los hongos.

$J l_{o}$ ^: sabroso ¡los hongos son sabrosos! Término utilizado para referirse a los hongos más apreciados por su sabor.

Jmeá güí: a humedad (oler), es común que los chinantecos digan que los hongos huelen a mojado ya que relacionan la presencia de estos por la humedad de los sitios de recolección.

Jmeá $t u^{\prime}$ : sabor a pollo, de acuerdo con algunos chinantecos existen especies de hongos que tienen sabor a pollo, como por ejemplo: Neolentinus suffrutescens (Brot.) T.W. May \& A.E. Wood.

Jmīo: raíz, término usado para designar el micelio basal de los esporomas.

Jти ий: amarillo, nombre del platillo elaborado a base de masa de maíz, puré de chile mezclado con tomate y hongos, el cuál es el más valorado por los habitantes de la población para preparar los hongos (Figura 2d).

Jnéë: amarillo, sufijo usado para nombrar especies de dicho color, tales son los casos de Cantharellus cibarius Fr., Amanita laurae Guzmán et Ramírez-Guillén y A. basii Guzmán et Ramírez-Guillén.

Jnёøö: ejote, sufijo utilizado para designar especies de color rosa o morado como Hygrophorus russula (Schaeff.) Kauffman e H. purpurascens (Alb. et Schwein.) Fr. en alusión al "ejote de cuarentena" o "pinto" (Phaseolus sp.); esta especie de leguminosa es uno de los principales alimentos cultivados por los habitantes de la comunidad.

Jnï: nube o niebla, sufijo utilizado para designar a las especies de hongos que presentan forma de nube o que salen en los bosques de niebla, por ejemplo Sparassis crispa (Wulfen) Fr. Jó: animal o ganado, sufijo utilizado para nombrar especies de hongos que crecen sobre o se asocian al estiércol de ganado, por ejemplo Agaricus campestris L.

Kuá: bendito, sufijo utilizado para nombrar especies del género Amanita sect. caesarea (tales como A. basii, A. jacksonii y A. laurae), y a Neolentinus suffrutescens, ya que son consi- derados como un alimento bendito en temporada de lluvias; este concepto está asociado con su buen sabor y con su abundancia.

Kuän: crecer, sufijo utilizado para nombrar especies que habitan sobre árboles o estiércol, tales como Agaricus campestris L., Pleurotus djamor (Rumph. ex Fr.) Boedijn y P. djamor var. roseus Corner.

Loguaa: oreja, sufijo utilizado para nombrar especies de hongos con forma de oreja de humanos, por ejemplo Auricularia polytricha (Mont.) Sacc. y A. delicata (Mont. ex Fr.) Henn.

Loguí: sombrero, término utilizado para nombrar el píleo de los hongos.

Mámä̈: hojarasca, término utilizado para nombrar el sustrato donde crecen algunas especies de hongos por ejemplo Laccaria amethysteo-occidentalis G.M. Muell., L. laccata (Scop.) Cooke, L. nobilis A.H. Sm. y L. trichodermophora G.M. Muell.

Maa dsie: árbol enfermo, estado de los árboles en el que algunas de especies de hongos parásitos se desarrollan, por ejemplo Ganoderma applanatum (Pers.) Pat.

Maa jgoo: árbol podrido, lugar donde crecen algunas especies de hongos saprobios, tales como Neolentinus suffrutescens.

Maa: árbol o palo, utilizado como sufijo para nombrar aquellas especies que crecen sobre árboles, tales como Neolentinus suffrutescens y Pleurotus djamor (Rump. ex Fr.) Boedijn.

'Majé: jonote, sufijo usado para nombrar a especies de hongos que crecen sobre dicha especie de árbol (Heliocarpus appendiculatus Turcz.), por ejemplo Pleurotus djamor.

'Makíri: pino, sufijo utilizado para nombrar especies de hongos que crecen únicamente en especies de árboles del género Pinus, por ejemplo Neolentinus suffrutescens.

'Matoo": platanal, sufijo utilizado para designar especies que crecen en dicho cultivo, por ejemplo Pleurotus djamor var. roseus Corner.

Mejée $e^{\wedge \wedge}$ tenate, recipiente de material natural utilizado para depositar los hongos recolectados en el monte.

Mú́: víbora, animal que algunos habitantes chinantecos han asegurado ver en visiones por el efecto provocado al consumir hongos alucinógenos.

Mic jü: semilla, término utilizado para nombrar el polvo constituido por las esporas de los hongos. 
Moóc: monte, relacionado con el ecosistema boscoso donde abundan las especies de hongos.

Motu': huevo, término utilizado para nombrar el estado inicial de desarrollo de algunas especies de hongos, principalmente dentro de la familia Amanitaceae.

Nai: hongo, término utilizado para nombrar a los hongos en Tlatepuzco, Oaxaca para el universo de los hongos (Skinner y Skinner, 2000).

'Náiñ : separar, acción llevada a cabo posterior a la colecta, y previa a cocinar los hongos, ya que de acuerdo a los chinantecos, las especies poseen diversas formas de preparación que optimizan su sabor.

Ngü̈: carne, término utilizado para nombrar el himenio y el contexto del hongo.

Ni cuu'tuziee": cola de guajolote, forma que han encontrado los chinantecos en algunas especies de hongos por ejemplo Ganoderma applanatum (Pers.) Pat. Esta característica la han utilizado en la elaboración de artesanías locales como portarretratos, los cuales cumplen una función ornamental en sus hogares (Fig. 2e).

Niii: sal, término utilizado para nombrar las escamas presentes en el píleo de algunas especies como por ejemplo Amanita muscaria (L.) Lam. con píleo rojo con escamas blancas o Amanita sp. con escamas piramidales blancas (Figura 2f).

Niur Nít: vender hongos, actividad realizada por algunos habitantes de la comunidad cuando existe abundancia de hongos.

Niti giú: hongo bueno, incluye el universo de los hongos comestibles.

Nít gui: hongo venenoso, usado para el universo de los hongos no comestibles.

Nít: hongo, se aplica como prefijo para cualquier nombre de hongos.

Noöㅁ: red o grasoso, sufijo utilizado para designar especies de hongos con consistencia grasosa o con himenio reticulado, por ejemplo Favolus tenuiculus P. Beauv.

'Nór Nít: buscar hongos, actividad realizada por gran parte de la población en temporada de fructificación de los hongos silvestres comestibles.

Niii: pasto o zacate, sufijo utilizado para nombrar especies que crecen sobre el pasto, por ejemplo Agaricus campestris L.

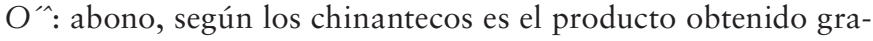
cias a la presencia de los hongos en el bosque.

Óo: viejo, sufijo utilizado para nombrar especies de hongos cuya fructificación puede durar varios meses o años sin degradarse, por ejemplo Ganoderma applanatum (Pers.) Pat. o Ganoderma spp.

Sde 'ma: sobre árboles, términos utilizados para designar a las especies de hongos que crecen sobre los árboles, por ejemplo Pleurotus djamor y P. djamor var. roseus.

Sd́́ guịi: hiel de ardilla, término usado para nombrar a las especies de hongos con forma o color verde como la hiel de ardilla, por ejemplo Craterellus tubaeformis (Fr.) Quél.

'Suoér: freír, forma favorita de preparar algunas especies de hongos, por ejemplo Hydnum spp. y Hebeloma fastibile (Pers.) P. Kumm.

Tá: miel, utilizada para conservar hongos alucinógenos del género Psilocybe, sustancia dulce mediante la cual dichos hongos prolongan sustancialmente su vida de anaquel (Figura 2g).

Tä́: pájaro, segundo sufijo utilizado para nombrar especies de hongos con estípite delgado que son semejantes a las patas de aves pequeñas, tales como especies del género Laccaria.

Táar ï: dibujar, actividad realizada por algunos habitantes, en la cual realizan dibujos sobre el himenio de especies tales como Ganoderma applanatum (Pers.) Pat. (Figura 2h).

Teé: blanco, sufijo utilizado para designar especies de hongos venenosos o blancos, tales como Amanita verna (Bull.) Lam. o A. virosa Bertill.

Tii: pie, término utilizado para nombrar el estípite de los hongos.

Toer'Nït: cortar hongos, forma de extraer los hongos de sus áreas naturales de producción durante la recolección.

Toloo: bolsa, contenedor utilizado para depositar las especies de hongos recolectados.

Toor': asar, forma favorita de preparar algunas especies de hongos tales como Amanita basii, A. jacksonii y A. laurae.

Tǘ: baboso, sufijo utilizado para designar a especies que presentan consistencia viscosa o pegajosa, por ejemplo el pilipelis de Suillus granulatus L. 
Tuziee : guajolote, utilizado para especies de hongos del género

Laccaria con estípite grueso, por ejemplo Laccaria trichodermophora G.M. Muell. y L. nobilis A.H. Smith.

Uü: chile, utilizado como sufijo para designar hongos de color rojo como Hypomyces lactifluorum (Schwein.) Tul. et C. Tul. en alusión al chile de árbol (Capsicum annum L.), el cual es ampliamente utilizado por los miembros de la cultura chinanteca.

Yöö: rojo, sufijo utilizado para designar especies que presenta coloración roja en cualquier parte del hongo, por ejemplo Amanita jacksonii Pomerl. en su píleo.

\section{DISCUSIÓN}

El registro de 70 vocablos relacionados con los hongos demuestra que actualmente el conocimiento tradicional, expresado a través de la riqueza lingüística, pervive en la cultura chinanteca. $\mathrm{Al}$ analizar los vocablos que componen los nombres de los hongos en chinanteco se aprecia que algunas especies pueden estar relacionadas con hasta cinco vocablos, lo que demuestra la riqueza de dicho idioma y la profundidad de conocimiento tradicional existente actualmente en personas mayores. En contraste, un dato importante en el estatus del conocimiento micológico en la comunidad se obtuvo en pláticas sobre el recurso micológico impartidas a alumnos de la escuela primaria de la comunidad donde se observó un gran desconocimiento de los hongos por parte de las nuevas generaciones. Esto se debe principalmente a la erosión de la identidad cultural y la occidentalización, tal como ocurre en otros grupos étnicos en donde estos factores han sido el punto de partida para la pérdida del conocimiento tradicional. Los nombres en chinanteco que reciben los hongos, son de cuatro tipos: i) binomiales, compuestos de dos vocablos, el primero haciendo referencia a la palabra hongo, mientras que el segundo a alguna característica o forma que presenta el hongo como por ejemplo Lactarius volemus var. volemus (Fr.) Fr. que tradicionalmente recibe el nombre de "Nít fii”(Nît: hongo; fii: leche); ii) trinomiales, son asignados de acuerdo a una característica particular parecida a un objeto o animal, asignando un adjetivo más específico para diferenciarlo de otros hongos que son parecidos, como el caso de Laccaria laccata var. pallidifolia y Laccaria nobilis nombrados como "Nít tii tä" (Nît: hongo; tii: pata; tä: pájaro), y "Nít tii tuziee"
(Nît: hongo; tii: pata; tuziee: guajolote), respectivamente; iii) tetranomiales, los nombres están compuestos por cuatro vocablos como en el caso de Auricularia spp. (incluyendo Auricularia polytricha (Mont.) Sacc., A. delicata y Auricularia sp.) denominada "Nít loguaa dsia ri" (Nît: hongo; loguaa : oreja; dsia: persona; ri: duende); iv) pentanomiales, los nombres están compuestos por cinco vocablos como el caso de Agaricus campestris "Nítkuän ni jmiï jo’” (Nît: hongo; kuän: crece; ni: sobre; jmiï: estiércol; jó: ganado) recibe este nombre debido a que crece sobre el estiércol de ganado, es importante mencionar que la traducción del último vocablo, puede referir, a los animales en general; sin embargo, las personas de la comunidad suelen llamar a su ganado con este término.

El estudio y análisis de las miconimias ha permitido la interpretación de la relación y el grado de conocimiento que el hombre tiene sobre una especie o un grupo de hongos. Es común que los recolectores asignen un término a varias especies que presentan características similares para facilitar el reconocimiento y clasificación de los hongos (Garibay-Orijel, 2009); estos nombres han sido denominado micotaxa. Es común que los nombres tradicionales y vocablos asignados a las especies comestibles tengan como base un término que significa hongo, acompañado de otro u otros haciendo referencia a cualidades del hongo o características del entorno que los rodea (GaribayOrijel et al., 2006). En algunas localidades la clasificación de los hongos separa a especies fúngicas de los hongos, es decir, algunos hongos son considerados como entidades diferentes o sin relación a los hongos, tal es el caso de las orejas (Auricularia spp. y Schizophyllum commune Fr.) que no son considerados como hongos por los habitantes de Teapa, Tabasco (Ruan-Soto et al., 2004). Mientras que el cuitlacoche (Ustilago maydis) no es considerado como un hongo por los habitantes chinantecos, sino como una estructura más de la milpa.

En algunos estudios etnomicológicos se ha reportado el cambio de nombres tradicionales por nombres en español (Garibay-Orijel, 2009; Ruan-Soto et al., 2004, 2009); sin embargo, en la comunidad de Santiago Comaltepec se mantiene el uso de los nombres locales, esto podría ser, debido al bajo impacto de aculturación española en la zona, por su aislamiento geográfico hasta fechas recientes, tal y como ocurre en otras partes del Estado de Oaxaca. Una situación similar se ha detectado en otros grupos 
étnicos mexicanos, por ejemplo en el grupo mixteco sobre todo en las comunidades que viven en regiones con aislamiento geográfico (Hernández-Santiago et al., 2017). Este hecho fundamenta el presente trabajo, dado que muestra la enorme importancia de documentar y preservar el conocimiento tradicional micofílico existente en la actualidad en la cultura chinanteca.

\section{CONCLUSIONES}

Se documenta por primera ocasión la riqueza lingüística relacionada con los hongos en la cultura chinanteca. De acuerdo a los vocablos registrados y la variedad de especies fúngicas utilizadas es posible asegurar que los hongos juegan un papel importante en la comunidad chinanteca estudiada. La percepción de los chinantecos del recurso micológico es muy amplia ya que la variedad conceptual de los nombres asignados permite afirmar que los hongos han sido conocidos por esta cultura desde épocas ancestrales, también es posible señalar que existen conceptos que fueron incorporados posteriormente. Prevalece una conservación del conocimiento tradicional de los hongos, expresada en la riqueza lingüística en idioma chinanteco que pervive actualmente; sin embargo, existe la posibilidad de que en un futuro estos nombres se pierdan o sean reemplazados por nombres en español, ya que por el proceso de transculturación el conocimiento tradicional se está perdiendo rápidamente. Este estudio de vocablos relacionados con el uso de los hongos, y los nombres en chinanteco asignados a los hongos, es el punto de partida para la realización de estudios etnomicológicos en la cultura chinanteca para tener una mayor aproximación de la percepción de este grupo hacia los hongos.

\section{AGRADECIMIENTOS}

Se agradece el apoyo financiero al proyecto CONACyT-24667.

\section{REFERENCIAS}

Anderson, L., D. Godwin, 2007. Leyendo y escribiendo el chinanteco de Santiago Comaltepec. Instituto Lingüístico de Verano, ciudad de México.

Arora, D., 1986. Mushrooms Demystified. 2nd edition. 9th printing. Ten Speed Press, Berkeley. Barbaras, M.A., A.M. Bartolomé, 1990. Historia chinanteca. CONACULTA. Oaxaca,México.

Burrola-Aguilar, C., O. Montiel, R. Garibay-Orijel, L. Zizumbo-Villarreal, 2012. Conocimiento tradicional y aprovechamiento de los hongos comestibles silvestres en la región de Amanalco, Estado de México. Revista Mexicana de Micología 35: 1-16.
Comisión Nacional de Población. (CONAPO), 1994. Los municipios de estado de Oaxaca (Sierra Norte). Anuario. Oaxaca, México.

Domínguez-Romero, D., R. Arzaluz, C.V. Valdés, P. Romero, 2015. Uso y manejo de hongos silvestres en cinco comunidades del municipio de Ocoyoacac, Estado de México. Tropical and Subtropical Agroecosystems 18: 133-143.

Garibay-Orijel, R., 2009. Los nombres zapotecos de los hongos. Revista Mexicana de Micología 30: 43-61.

Garibay-Orijel, R., J. Cifuentes, A. Estrada-Torres, J. Caballero, 2006. People using macro-fungal diversity in Oaxaca, México. Fungal Diversity 21: 41-67.

Gordon, R., 2005. Ethnologue: Languages of the World. SIL International, Dallas.

Guzman, G. 1983. The Genus Psilocybe: A systematic revision of the known species including the history, distribution and chemistry of the Hallucinogenic Species. Nova Hedwigia 74. Cramer, Vaduz.

Guzmán, G., 2011. El uso tradicional de los hongos sagrados: pasado y presente. Etnobiología 9: 1-21.

Guzmán G., F. Ramírez-Guillén, 2001. The Amanita caesarea-complex. Bibliotheca Mycologica 187: 1-66.

Hernández Santiago, F., J. Pérez Moreno, B. Xoconostle Cázares, J.J. Almaraz Suárez, E. Ojeda Trejo, G. Mata, I. Díaz Aguilar, 2016. Traditional knowledge and use of wild mushrooms by Mixtecs or $\tilde{N} u u s a v i$, the people of the rain, from Southeastern Mexico. Journal of Ethnobiology and Ethnomedicine 12: 35.

Instituto Nacional Indigenista (INI), 2003. Consultado el 12 de enero de 2016. Disponible en http://www.ini.gob.mx.

Instituto Nacional de Estadística y Geografía (INEGI), 2010. Censo poblacional. (Santiago Comaltepec, Oaxaca), Oaxaca, México.

Instituto Nacional de Estadística y Geografía (INEGI), 2015. Censos y conteos de población y vivienda, Oaxaca, México.

Pérez-Moreno, J., 2012. Los hongos comestibles ectomicorrízicos y su biotecnología. In: Sánchez, J.E., G. Mata (eds.), Hongos comestibles y medicinales de Iberoamérica: investigación y desarrollo en un entorno multicultural. Colegio de la Frontera Sur - Instituto de Ecología, Tapachula, Chiapas. Pp. 19-28.

Pérez-Moreno, J., M. Martínez-Reyes, 2014. Edible ectomycorrhizal mushrooms: biofactories for sustainable development. In: Guevara-González, R, I. Torres-Pacheco (eds.), Biosystems engineering: Biofactories for food production in the century XXI. Springer International Publishing, Basel, Switzerland. Pp. 151233.

Ruan-Soto, F., R. Garibay-Orijel, J. Cifuentes, 2004. Conocimiento micológico tradicional en la planicie costera del Golfo de México. Revista Mexicana de Micología19: 57-70.

Ruan-Soto, F., J. Cifuentes, R. Mariaca, F. Limón, L. Pérez-Ramírez, S. Sierra, 2009. Uso y manejo de hongos silvestres en dos comunidades de la Selva Lacandona, Chiapas, México. Revista Mexicana de Micología 29: 61-72.

Rupp J., H. Mendoza-Salas, L. Carillo-Mendoza, 2012. Diccionario chinanteco para Ayotzintepec, Ozumacín y Progreso. Instituto Lingüístico de Verano, Ciudad de México.

Rzedowski, J., 2006. Vegetación de México. Comisión Nacional para el Conocimiento y Uso de la Biodiversidad. México. 1ra. Edición digital. Comisión Nacional para el Conocimiento y Uso de la Biodiversidad, Ciudad de México.

Sandoval, C., 2002. Investigación cualitativa. Programa de especialización en teoría, métodos y técnicas de investigación social. ICFES, Bogotá. Colombia.

Skinner, L., M. B. Skinner, 2000. Diccionario chinanteco de San Felipe Usila, Oaxaca. Instituto Lingǘstico de Verano, Ciudad de México.

Trudell, S., J. Ammirati, 2009. Mushrooms of the Pacific Northwest. Timber Press, Portland. 